\title{
Efeitos da meditação associada a educação em neurociências da dor em adultos com fibromialgia: ensaio clínico controlado e randomizado*
}

\author{
Lucas Pelegrini Nogueira de Carvalho ${ }^{1,2}$ \\ (D) https://orcid.org/0000-0002-5027-2042 \\ Lílian Silva Araújo² \\ (iD) https://orcid.org/0000-0002-6267-7573 \\ Maria do Perpétuo Socorro Lage Avelar ${ }^{3}$ \\ (D) https://orcid.org/0000-0002-4061-7908 \\ Karina Gramani Say ${ }^{2}$ \\ (iD) https://orcid.org/0000-0002-2451-8109
}

Objetivo: analisar os efeitos do mindfulness associado com educação em neurociências da dor na percepção da dor, sono e capacidade funcional de adultos com fibromialgia. Método: trata-se de um estudo experimental controlado e randomizado cujos participantes foram divididos em dois grupos: grupo mindfulness e grupo mindfulness e educação em neurociência da dor. Resultados: foram observadas melhorias de algumas variáveis relacionadas à dor, na capacidade funcional, ansiedade, depressão, qualidade do sono e na catastrofização. Não houve diferença na comparação intergrupos, porém existiram diferenças na comparação intra-grupos. Conclusão: intervenções baseadas na prática de mindfulness e educação em neurociências da dor se mostraram eficazes na melhora do quadro de pacientes com fibromialgia. Registro do Ensaio clínico: REBEC RBR-58pgs4.

Descritores: Idoso; Dor Crônica; Fibromialgia; Sono; Atenção Plena; Educação em Saúde.

\section{Como citar este artigo}

Pelegrini LNC, Araújo LS, Avelar MPSL, Say KG. Effects of meditation associated with education in neurosciences of pain in adults with fibromyalgia: A randomized controlled trial. SMAD, Rev Eletrônica Saúde Mental Álcool Drog. 2020;16(3):3-13. doi: https://dx.doi.org/10.11606/issn.1806-6976.smad.2020.167602 


\title{
Effects of meditation associated with education in neurosciences of pain in adults with fibromyalgia: A randomized controlled trial
}

\begin{abstract}
Objective: to analyze the influence of meditation associated with pain neuroscience education in pain perception, sleep and functional capacity of adults with fibromyalgia. Method: this is an experimental, controlled, randomized study whose participants were divided into two groups: mindfulness group and mindfulness group and education in pain neuroscience. Results: improvements were observed in some variables related to pain, functional capacity, anxiety, depression, sleep quality and catastrophization. 270/5000. There was no difference in the intergroup comparison, but there were differences in the intra-group comparison. Conclusion: mindfulness-based interventions and education in pain neurosciences proved to be effective in improving the condition of patients with fibromyalgia. Clinical trial register: REBEC RBR-58pgs4
\end{abstract}

Descriptors: Elderly; Aging; Chronic Pain; Fibromyalgia; Sleep; Mindfulness; Health Education.

\section{Efectos de la meditación asociada con la educación en neurociencia del dolor en adultos con fibromialgia: un ensayo clínico aleatorio controlado}

\begin{abstract}
Objetivo: analizar los efectos de la atención plena asociada con la educación en neurociencias del dolor sobre la percepción del dolor, el sueño y la capacidad funcional de los adultos con fibromialgia. Método: este es un estudio experimental, controlado, aleatorizado cuyos participantes se dividieron en dos grupos: grupo de atención plena y grupo de atención plena y educación en neurociencia del dolor. Resultados: se observaron mejoras en algunas variables relacionadas con el dolor, la capacidad funcional, la ansiedad, la depresión, la calidad del sueño y la catastrofización. No hubo diferencias en la comparación intergrupal, pero sí hubo diferencias en la comparación intragrupo. Conclusión: las intervenciones basadas en la práctica de la atención plena y la educación en neurociencias del dolor demostraron ser efectivas para mejorar la condición de los pacientes con fibromialgia. Registro del ensayo clínico: REBEC RBR-58pgs4
\end{abstract}

Descriptores: Ancianos; Dolor Crónico; Fibromialgia; Sueño; Atención Plena; Educación para la Salud. 


\section{Introdução}

O mundo e o Brasil têm passado por um intenso envelhecimento populacional(1-2). Como consequência das alterações na estrutura etária da população e aos avanços nas ciências da saúde, pode-se observar o fenômeno denominado transição epidemiológica. O mesmo se refere ao processo de redução das doenças infectocontagiosas e ao aumento de doenças crônicodegenerativas ${ }^{(1)}$. As doenças crônicas interferem na capacidade funcional dos indivíduos acometidos, além de prejudicar a independência e na qualidade de vida dos indivíduos idosos ${ }^{(3)}$. Além disso, muitos quadros vêm acompanhados de dor, sendo a dor crônica uma das principais queixas ${ }^{(4)}$.

A Associação Internacional de Estudos da Dor (IASP) classifica a dor como "experiência desagradável, sensitiva e emocional, associada ou não ao dano real ou potencial de lesões dos tecidos e relacionada com a memória individual, com as expectativas e as emoções de cada pessoa", podendo ser dividida entre aguda que ocorre de forma súbita - ou crônica - duração de 6 meses ou mais.

A prevalência de dor crônica na população brasileira é de 29,3 a $73,3 \%{ }^{(5)}$. Tal prevalência é um terço maior em mulheres do que em homens; além disso, é mais prevalente entre indivíduos com mais de 65 anos quando comparada àqueles com menos de $44^{(6)}$. Apesar de não ser um fator normativo da senescência, a dor crônica está associada a doenças crônico-degenerativas que podem aparecer nesse período da vida(7-9).

Alguns desfechos indesejáveis da dor crônica se relacionam não apenas à percepção negativa de saúde, como também e no bem-estar psicológico, biológico e espiritual(10). Outras condições advindas no processo da dor crônica seriam a dependência nas atividades de vida diária, limitações no convívio social, mudanças na sexualidade, desequilíbrio econômico, desesperança e depressão, fatores que, ao se somarem, interferem diretamente na qualidade de vida(4,11-13).

Nesse contexto, a fibromialgia, uma doença caracterizada por dor musculoesquelética difusa e crônica também associada a outros distúrbios psicológicos, como ansiedade, depressão e sono, está altamente associada a quadros de insônia crônica e sono não restaurador - 76 a 90\% dos fibromiálgicos relatam sono não reparador, enquanto essa prevalência é de 10 a $30 \%$ em indivíduos normais ${ }^{(14)}$.

Muitos fatores contribuem para a aparição da doença - genéticos, infecções, doenças autoimunes, estilo de vida ou abalos emocionais- mas, a alteração neural provocada pelo comportamento nocivo deve ser destacada, pois gera maior conectividade cerebral nas regiões que são responsáveis pela modulação e avaliação de estímulos nocivos e áreas responsáveis também pela consciência interceptiva e sensações corporais, o que tem por consequência uma maior sensibilização nos processos nociceptivos e gera a cronificação dos processos dolorosos ${ }^{(14-15)}$. A literatura revela que a prevalência da fibromialgia varia entre 0,2 e 6,6\% da população, oscilando de 2,4 a $6,8 \%$ entre as mulheres (16).

Diante do presente cenário, estudos sugerem que intervenções baseadas em mindfulness demonstram bons resultados no tratamento de doenças como fibromialgia, transtornos de ansiedade, câncer, hipertensão e entre outras doenças, uma vez que melhora nos sintomas como a dor, ansiedade e depressão, além de promover a sensação de bem-estar ${ }^{(11,17-18)}$.

Mindfulness pode ser definido como uma prática que integra a mente e o corpo visando, principalmente, a redução do estresse, mas acima de tudo a orientação da consciência para o momento presente e sem julgamentos $^{(19)}$. Outros autores ainda trazem que o processo de meditação focado em atenção plena é estar atento aos processos que ocorrem no momento presente, contemplando experiências com atitudes de curiosidade em nuanças sutis do cotidiano, sentir, perceber e aceitar os fatos cotidianos de modo não reativo estando em controle com o nosso cérebro, conectividade e controle do próprio bem-estar subjetivo(20).

A prática de meditação pode ser uma intervenção terapêutica segura, eficaz e efetiva, promovendo a saúde e melhorias na qualidade de vida, propiciando: a melhora na aceitação da dor, melhor manejo de situações estressantes, diminuição da agressividade em pacientes psiquiátricos e melhora na qualidade de vida e auto eficácia(19). Isso porque a meditação proporciona a redução da atividade do sistema nervoso simpático, induzindo uma menor ativação neural, o que faz reduzir o trabalho dos mecanismos cerebrais que são responsáveis pela sensibilização das regiões o cérebro por exercerem influencias nos processos dolorosos ${ }^{(21)}$, e por consequência o aumento da ação do sistema nervoso parassimpático, refletindo em um melhor funcionamento de funções neuroendócrinas, metabólicas e neurais, aumentando a concentração de melatonina, serotonina, ácido gama-aminobutírico (GABA) e a diminuição de cortisol e norepinefrina ${ }^{(15)}$.

Embora crescente o interesse em formas alternativas de tratamentos, estudos sobre meditação encontram-se escassos, especialmente com a população idosa. Existem evidências de que os efeitos analgésicos da prática meditativa podem apresentar significância a partir de oito semanas de intervenção(22).

Além disso, a falta de conhecimento por parte dos pacientes sobre sua doença pode resultar em um empecilho no seu tratamento, por se criar comportamentos e estratégias mal adaptativas para 
o enfrentamento da dor, como a hipervigilância e a desassociação dos comportamentos de estilo de vida com o quadro de dor ${ }^{(22-23)}$. Acredita-se que a prática de Educação em Neurociências da Dor (END) pode contribuir para uma melhor classificação de dor na perspectiva dos pacientes no que tange sobre o conhecimento da dor, pensamento catastrófico, prevenção do medo, depressão, ansiedade, estresse, atitudes e comportamentos da dor e utilização dos serviços de saúde(24-26). O objetivo da terapêutica é descrever como o sistema nervoso age por meio da sensibilização dos nervos periféricos e centrais e como o processamento cerebral interpreta as informações dos tecidos e a ativação neural, que resultam na capacidade de modelar a dor e que nem sempre essa representação se dá de forma verdadeira(27).

A END, por se tratar de um método de boa resolubilidade e baixo custo passa a ser uma alternativa para a economia do sistema público de saúde. Nessa perspectiva, a utilização desse método vem sendo uma estratégia promissora para o enfrentamento dos problemas que hoje encontram-se na área da saúde, evidenciando cada vez mais a troca bidirecional de saberes, na qual o profissional detentor de conhecimento técnico passa esse conhecimento para o paciente e o mesmo traz alternativas populares mais próximas a realidade para lidar com sua problemática.

Sendo assim, este estudo teve por objetivo verificar os efeitos da prática de Mindfulness associada à prática de educação em neurociência da dor na percepção da dor de adultos diagnosticados com fibromialgia residentes em um município do interior paulista. Além desta variável, foram analisados os efeitos destas intervenções para o sono, humor e capacidade funcional da amostra estudada.

\section{Método}

\section{Participantes e local do estudo}

Trata-se de um ensaio clínico controlado e randomizado, cujos participantes foram pessoas com idade maior ou igual a 40 anos diagnosticadas com fibromialgia e atendidas no Sistema Único de Saúde do município de São Carlos, São Paulo, Brasil. Os indivíduos foram aleatoriamente alocados em dois grupos: Grupo Mindfulness e Educação em Neurocência da Dor (GMEND), cujos participantes passaram por ambas intervenções e no grupo controle ativo, cuja intervenção foi a prática de mindfulness (GM).

Os critérios de inclusão foram: ter idade maior ou igual a 40 anos, possuir o diagnóstico médico de fibromialgia, com intensidade da dor classificada em moderada ou grave por meio Escala de Numérica de Classificação da Dor, sendo 0 considerada sem dor, de
1 a 3 dor leve, de 4 a 6 dor moderada e de 6 a 10 dor severa. Foram excluídos os indivíduos que apresentaram indício de declínio cognitivo ao pontuar abaixo da nota de corte no Mini Exame do Estado Mental (MEEM) - no caso de idosos participantes; ter limitações no quadril ou joelho, ou dificuldades de realizar movimentos no nível do chão; praticar atividade física regularmente, correspondendo a mais que 150 minutos de atividade física semanalmente; ter algum acometimento emocional agudo (acontecimento nos últimos 5 meses que o deixe emocionalmente vulnerável, como por exemplo mortes, acidentes e outras questões de ordem pessoal e emocional); auto relato de transtorno obsessivo compulsivo (TOC); estar em tratamento de fibromialgia ou submetido a tal em um prazo inferior a 3 meses. Além disso, foram descontinuados do estudo os participantes que apresentaram mais de $25 \%$ de faltas nas intervenções (o equivalente a 3,25 faltas).

\section{Instrumentos, procedimentos de coleta e análise dos dados}

Ocorreram duas avaliações (pré e pós-intervenção) em dia e horário previamente agendados. As mesmas ocorreram na Unidade de Saúde Escola da Universidade Federal de São Carlos - UFSCar, em ambiente reservado e livre de interferências. Os avaliadores foram membro do grupo LADORFE (Laboratório de Estudos em Dor e Funcionalidade no Envelhecimento). Para a coleta de dados, foram utilizados: um questionário sociodemográfico, especificamente elaborado para esse estudo, seguido de uma escala numérica para a classificação da intensidade da dor, avaliação do estado mental pelo Mini Exame do Estado Mental (MEEM) e questões específicas do critério de exclusão, perguntas referentes a intensidade, localização e impacto da dor por meio do Inventário Breve de Dor, avaliação da capacidade funcional por meio da Escala de Independência em Atividades de Vida Diário de Katz e Avaliação das Atividades Instrumentais de Vida Diária de Lawton, avaliação da qualidade do sono pela Escala de Pittsburgh, rastreio de sintomas depressivos pela Escala de Depressão em Geriatria (GDS), um questionário para avaliar o impacto da fibromialgia na vida do paciente (FIQ- impacto da fibromialgia), uma escala de avaliação de ansiedade (Escala Hospitalar de Ansiedade e Depressão- HAD, avaliação do grau de catastrofização quanto à ruminação, desamparo aprendido e magnificação por meio da Escala de Catastrofização de Dor, avaliação da cinesiofobia e a abordagem da dor e sua intensidade por meio da Escala Tampa para Cinesiofobia (ETC).

Os dados foram analisados por meio da análise estatística descritiva e quantitativa em comparação intragrupos e intergrupos. Primeiramente, para verificar a 
normalidade, todos os dados foram submetidos ao teste de Shapiro-Wilks. Posteriormente, o Teste U-Mann-Whitney foi utilizado para análise intergrupo e Wilcoxon para análise intragrupos. O nível de significância para ambos foi de $p$ $\leq$ 0,05 e, para as análises, foi utilizado o STATISTICA 7.0.

\section{Intervenção}

O GMEND foi submetido as aulas de Educação em Neurociências da Dor, cujo foco era aprender a neurobiologia e neurofisiologias dos processos dolorosos. Os encontros de END tiveram duração de 30 minutos cada e ocorreram por 3 semanas. Terminado o protocolo da END, o GMEND foi então submetido às práticas de mindfulness, que foram realizadas na Unidade Saúde Escola - USE, localizada dentro da Universidade Federal de São Carlos - UFSCar, em parceria com instrutoras capacitadas para o desenvolvimento do programa de mindfulness. Os encontros ocorreram duas vezes por semana, com duração de 1 hora, durante 13 sessões. O GM atendeu aos mesmos critérios e protocolos que foram aplicados no GMEND, as práticas meditativas tiveram as mesmas características e duração, entretanto esse grupo não foi submetido as aulas de Educação em Neurociências da Dor.

A intervenção em Educação em Neurociências da Dor teve por objetivo estabelecer uma ação educativa no qual pudesse apresentar a neurobiologia dos processos dolorosos e fazendo compreender e tirar as principais dúvidas, o porquê eles ocorrem e quais medidas ajudam ou pioram o quadro do paciente com dor crônica. A intervenção foi aplicada por um membro do LADORFE, que foi capacitada para a aplicação da intervenção.

Para a prática de mindfulness, foi elaborado um protocolo adaptado da prática de mindfulness para redução de estresse (MBSR) ${ }^{(28)}$. O protocolo consistiu em uma prática em 13 sessões com duração de 1 hora para cada encontro. Devido às características dos participantes, nesta adaptação do protocolo não houve o dia de imersão. A prática se baseou no treinamento da mente e aspectos da atenção ancoradas em alguma atividade cognitiva ou a própria respiração. A técnica também consistia no treino de deixar de ser menos reativos a emoções negativas do dia a dia bem como condicionar os momentos de felicidade a pequenos prazeres e aprendendo a estimular a gratidão e compaixão. Ressalvamos que as professoras possuíam qualificação para aplicar o método, e durante o estudo foram voluntárias cegas, isto é, são sabiam quais dos participantes tinham realizado as aulas de Educação em Neurociências da Dor.

\section{Aspectos éticos}

Foram respeitadas as exigências da Resolução 466/2012 regulamentada pelo Conselho Nacional de Saúde, que trata de pesquisas que envolvem seres humanos. Fizeram parte deste estudo somente indivíduos adultos e idosos que concordaram em participar e assinaram o Termo de Consentimento Livre e Esclarecido. Esse estudo foi aprovado ao Comitê de Ética em Pesquisa de Seres Humano da Universidade Federal de São Carlos (no do parecer: 2.848.109).

\section{Resultados}

Na Figura 1, apresenta-se o fluxograma sobre como se deu o andamento deste estudo. A intervenção ocorreu em dois momentos: um no segundo semestre de 2018 e outro no início do primeiro semestre em 2019. O recrutamento de participantes se deu por meio da divulgação em mídias sociais como por exemplo o Facebook, Radio UFSCar, jornais da cidade, panfletos, cartazes, divulgação oral em salas de espera de Unidades Básicas de Saúde (UBS). No primeiro momento avaliamos 15 voluntários que manifestaram interesse na participação do projeto, sendo que 9 deles foram incluídos por atenderem os critérios da pesquisa. Destes, 5 foram ou por desistirem ou por excederem o limite de faltas estipulados no momento da intervenção. Na segunda parte do estudo, 92 indivíduos manifestaram interesse para participar; 39 deles foram avaliados e 36 foram incluídos. Ao final, 21 voluntários foram descontinuados do estudo ou por desistirem ou por excederem o limite de faltas estipulados no momento da intervenção. Os motivos pelos quais alguns voluntários desistiram foram: problemas de saúde, tanto do participante como de familiares dos participantes, adversidades ambientais como a dificuldade de transporte e/ou financeira para chegar no local onde a pesquisa foi realizada, além da não familiaridade com a prática, que, por consistir em um treino mental e uma capacidade cognitiva aprendida, leva em considerações aspectos subjetivos e intrínsecos dos participantes.

Os dados das integrantes do GMEND $(n=7)$ são mostrados na Tabela 1. Todas as participantes eram do sexo feminino, com média de idade igual a 58,5 anos ( $\pm 12,84)$, com pontuação média de $7,5( \pm 1,98)$ na Escala Numérica de Classificação da dor. Cinco das participantes eram casadas, uma solteira e uma viúva; duas possuíam ensino médio incompleto, duas tinham ensino médio completo, duas com ensino fundamental completo e uma com ensino superior completo. No GM $(n=12)$, onze participantes eram do sexo feminino. A média de idade desse grupo foi de 54 anos $( \pm 7,22)$, e a pontuação média na Escala Numérica de Classificação da dor igual a 6,25 ( $\pm 1,71)$. Dos participantes, oito eram casados, dois eram solteiros e dois eram divorciados. Com relação à escolaridade, dois possuíam ensino fundamental incompleto, três possuíam ensino médio completo, cinco possuíam ensino superior completo e dois possuíam pós-graduação. Os dados do GM são apresentados na Tabela 1. 


\section{Fluxograma de pesquisa}

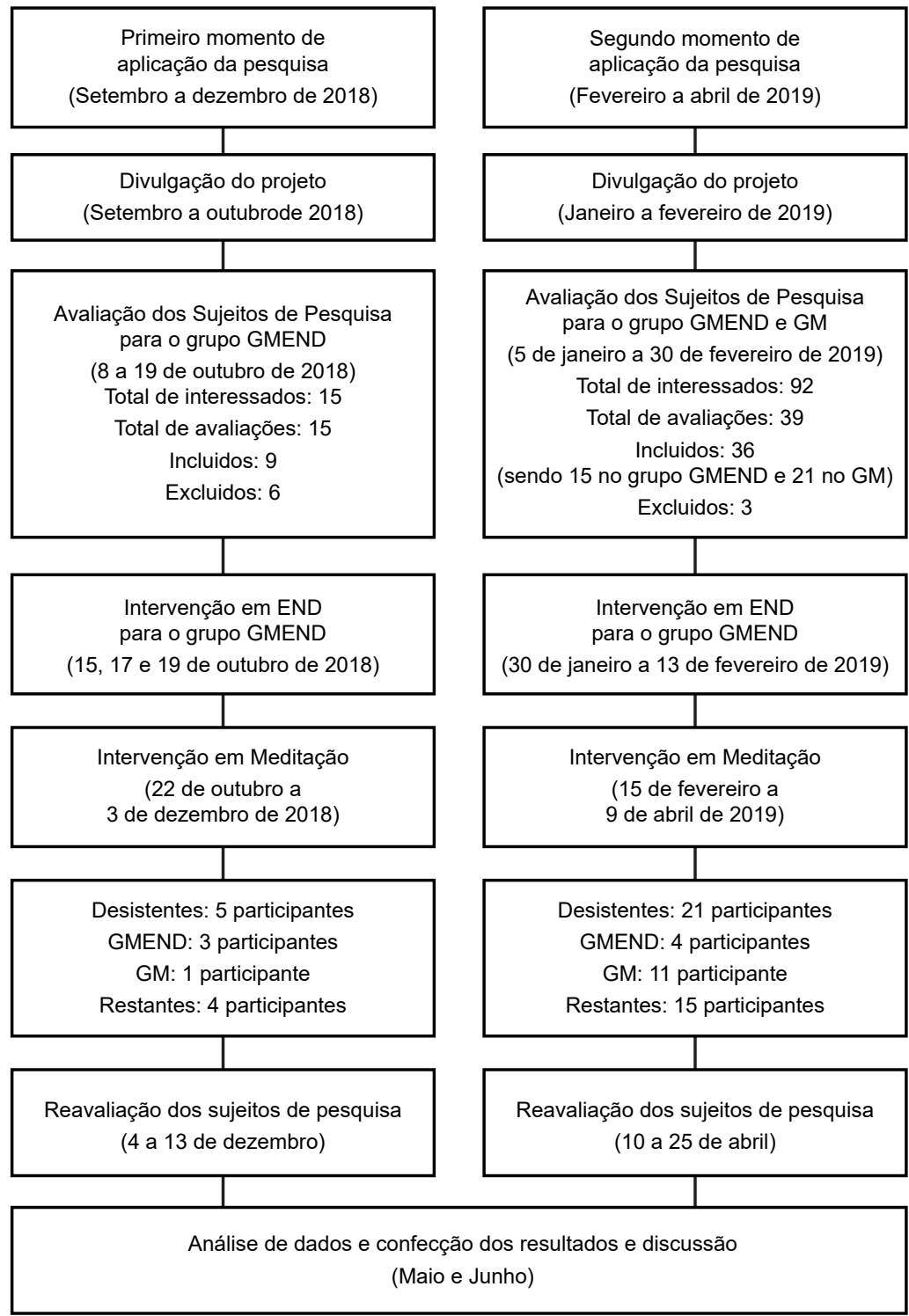

*GMEND - Grupo Mindfulness e Educação em Neurocência da Dor; ${ }^{\dagger} \mathrm{GM}=$ Grupo de meditação

Figura 1 - Fluxograma do desenvolvimento da pesquisa, São Carlos, SP, Brasil, 2020

Tabela 1 - Caracterização do GMEND*. São Carlos, SP, Brasil, 2020

\begin{tabular}{|c|c|c|c|c|c|c|c|}
\hline Código & Idade (anos) & Sexo & Escolaridade & $\begin{array}{c}\text { Estado } \\
\text { civil }\end{array}$ & $\begin{array}{l}\text { Escala Numérica de } \\
\text { Classificação da dor }\end{array}$ & Escala Katz & Escala Lawton \\
\hline C1 & 70 & $\mathrm{Fem}^{\dagger}$ & $\mathrm{EFC}^{\ddagger}$ & Solteira & 9 & $\begin{array}{c}\text { Dependente em } \\
1 \text { ABVD }\end{array}$ & Dependente Parcial \\
\hline $\mathrm{C} 2$ & 51 & $\mathrm{Fem}^{\dagger}$ & EMI" & Casada & 9 & - & - \\
\hline $\mathrm{C} 3$ & 81 & $\mathrm{Fem}^{\dagger}$ & EMI" & Viúva & 7 & $\begin{array}{c}\text { Dependente em } \\
1 \mathrm{ABVD}^{\S}\end{array}$ & Independente \\
\hline $\mathrm{C} 7$ & 52 & $\mathrm{Fem}^{\dagger}$ & EMC $\pi$ & Casada & 8 & - & - \\
\hline $\mathrm{C} 8$ & 61 & Fem. & $\mathrm{ESC}^{* *}$ & Casada & 5 & $\begin{array}{c}\text { Dependente em } \\
1 \mathrm{ABVD}^{\S}\end{array}$ & Independente \\
\hline $\mathrm{C} 14$ & 49 & Fem. & $\mathrm{EFC}^{\ddagger}$ & Casada & 5 & - & - \\
\hline C15 & 46 & Fem. & EMCף & Casada & 10 & - & - \\
\hline Código & Idade (anos) & Sexo & Escolaridade & $\begin{array}{c}\text { Estado } \\
\text { civil }\end{array}$ & Escala Katz & & Escala Lawton \\
\hline $\mathrm{C} 4$ & 57 & $\mathrm{Fem}^{\dagger}$ & $\mathrm{EFI}^{\dagger}$ & Casada & - & & - \\
\hline C5 & 57 & $\mathrm{Fem}^{\dagger}$ & $\mathrm{ESC}^{* *}$ & Casada & - & & - \\
\hline $\mathrm{C} 6$ & 50 & $\mathrm{Fem}^{\dagger}$ & $\mathrm{ESC}^{* *}$ & Casada & - & & - \\
\hline
\end{tabular}


Tabela 1 - continuação

\begin{tabular}{|c|c|c|c|c|c|c|}
\hline Código & Idade (anos) & Sexo & Escolaridade & $\begin{array}{c}\text { Estado } \\
\text { civil }\end{array}$ & Escala Katz & Escala Lawton \\
\hline $\mathrm{C9}$ & 57 & $\mathrm{Fem}^{\dagger}$ & $P G \S$ & Solteira & - & - \\
\hline C10 & 54 & $\mathrm{Fem}^{\dagger}$ & EMC $\pi$ & Casada & - & - \\
\hline C11 & 53 & $\mathrm{Fem}^{\dagger}$ & EMC $\pi$ & Casada & - & - \\
\hline C12 & 44 & $\mathrm{Fem}^{\dagger}$ & EMC $\pi$ & Casada & - & - \\
\hline C13 & 59 & $\mathrm{Fem}^{\dagger}$ & $\mathrm{ESC}^{\star *}$ & Divorciada & - & - \\
\hline C16 & 64 & $\mathrm{Fem}^{\dagger}$ & $\mathrm{ESC}^{* *}$ & Divorciada & Independente em todas as ABVD $§$ & Independente \\
\hline $\mathrm{C} 17$ & 64 & $\mathrm{Fem}^{\dagger}$ & $\mathrm{EFI+ \dagger}$ & Solteira & $\begin{array}{c}\text { Independente } \\
\text { Em todas as ABVD\$ }\end{array}$ & Independente \\
\hline C18 & 48 & Mas $\ddagger$ & $\mathrm{ESC}^{* *}$ & Casado & & - \\
\hline C19 & 41 & $\mathrm{Fem}^{\dagger}$ & $P G \S \S$ & Casada & & - \\
\hline
\end{tabular}

*GMEND = Grupo Meditação e Neurociência da Dor; ${ }^{\dagger} \mathrm{FEM}=$ Feminino; ${ }^{\star}$ EFC = Ensino Fundamental Completo; ${ }^{\circledR}$ ABVD $=$ Atividades Básicas de Vida Diária Observação que nem todos os pacientes eram idosos, portanto nem todos foram submetidos aos instrumentos desenvolvidos para a população idosa; "EMI = Ensino Médio Incompleto; ${ }^{~} \mathrm{EMC}=$ Ensino Médio Completo; ${ }^{* *} \mathrm{ESC}=$ Ensino Superior Completo; ${ }^{+\dagger}$ EFI = Ensino Fundamental Incompleto; ${ }^{\ddagger \neq}$ Masc $=$ Masculino; ${ }^{\S}$ PG = Pós-Graduação

Observou-se diferença significativa entre os grupos no momento pré-intervenção na variável medida pelo Questionário de Impacto da Fibromialgia - FIQ $(p=0,02)$, porém não na avaliação pós-intervenção. A Tabela 2 mostra os resultados das avaliações prée pós-intervenção e os valores de $p$ para as análises conduzidas. Pode-se observar que os grupos não tiveram diferenças significativas e que os resultados as avaliações pós-intervenção tiveram uma melhor expressão.

No Inventário Breve de Dor encontrou-se diferença significativa para as variáveis "pior dor nas últimas 24 horas" $(p=0,03)$, "média de dor" $(p=0,04)$ e "interferência da dor no humor" $(p=0,02)$. A Tabela 4 mostra os valores pré- e pós-intervenção para o instrumento supracitado.

Foram também realizadas análises intragrupos. Como apresentado na tabela a seguir, o grupo GMEND apresentou diferenças significativas nas variáveis pontos de $\operatorname{dor}(p=0,017)$, na variável de dor mais leve nas últimas 24 horas ( $p=0,017)$, na variável interferência da dor nas atividades gerais $(p=0,027)$ e na variável interferência da dor no sono $(p=0,02)$ para o Inventário Breve de Dor. Houve também diferença no resultado do FIQ $(p=0,017)$ e nas variáveis de catastrofização e cinesiofobia $(p=0,017)$. A tabela 4 mostra os resultados da análise pré- e pós-intervenção para o GMEND. Com relação à análise do GM, observou-se diferença significativa para as variáveis pontos de dor $(p=0,002)$, interferência da dor nas atividades gerais $(p=0,018)$, interferência da dor no humor $(p=0,034)$, interferência da dor na caminhada $(p=0,024)$, interferência da dor no trabalho $(p=0,003)$, interferência da dos nos relacionamentos $(p=0,010)$ e interferência da dor no sono $(p=0,005)$, do no Inventário Breve de Dor, assim como no questionário de impacto da fibromialgia $(p=0,007)$, nos sintomas de ansiedade $(p=0,001)$ e depressão $(p=0,02)$. A Tabela 4 mostra os resultados das avaliações para o GM.

Tabela 2 - Comparação do desempenho do grupo meditação e neurociência da dor e grupo meditação nas avaliações pré e pós-intervenção. São Carlos, SP, Brasil, 2020

\begin{tabular}{|c|c|c|c|c|c|c|}
\hline \multirow{3}{*}{ Instrumentos de avaliação } & \multicolumn{6}{|c|}{ Grupos } \\
\hline & \multicolumn{3}{|c|}{ Pré avaliação } & \multicolumn{3}{|c|}{ Pós avaliação } \\
\hline & GMEND* & $\mathrm{GM}^{\dagger}$ & $p$ & GMEND* & $\mathrm{GM}^{\dagger}$ & $P$ \\
\hline $\mathrm{FIQ} \ddagger$ & $69,42 \pm 8,75$ & $55,44 \pm 13,01$ & 0,02 & $31,95 \pm 18,94$ & $33,42 \pm 20,85$ & 0,75 \\
\hline$H A D^{\S}$ & $8,57 \pm 1,90$ & $10,75 \pm 3,72$ & 0,14 & $5,85 \pm 3,80$ & $7,33 \pm 4,03$ & 0,65 \\
\hline Ansiedade & $7,85 \pm 5,52$ & $8,5 \pm 3,23$ & 0,55 & $3 \pm 3,55$ & $6,41 \pm 3,72$ & 0,06 \\
\hline Depressão & $13,42 \pm 2,57$ & $11,41 \pm 5,45$ & 0,58 & $6,57 \pm 3,69$ & $7,08 \pm 4,39$ & 0,96 \\
\hline Qualidade de Sono & $27,28 \pm 9,46$ & $23,66 \pm 12,69$ & 0,75 & $12,85 \pm 9,80$ & $11,33 \pm 9,34$ & 0,71 \\
\hline Catastrofização & $43 \pm 7,34$ & $41,36 \pm 9,03$ & 0,61 & & & \\
\hline
\end{tabular}

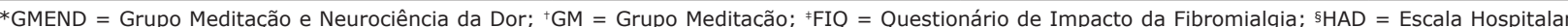
de Ansiedade e Depressão 
Tabela 3 - Comparação do desempenho dos grupos GMEND* e $\mathrm{GM}^{+}$para o Inventário Breve de Dor nas avaliações pré e pós-intervenção. São Carlos, SP, Brasil, 2020

\begin{tabular}{|c|c|c|c|c|c|c|}
\hline & \multicolumn{6}{|c|}{ Grupos } \\
\hline & \multicolumn{3}{|c|}{ Antes } & \multicolumn{3}{|c|}{ Depois } \\
\hline & GMEND* & $\mathbf{G M}^{\dagger}$ & $P$ Valor & GMEND* & $\mathbf{G M}^{\dagger}$ & $P$ Valor \\
\hline Pontos de dor & $40,42 \pm 5,12$ & $11 \pm 8,28$ & 0,22 & $36,91 \pm 11,01$ & $13,16 \pm 11,91$ & 0,82 \\
\hline Pior dor (24h) & $8,57 \pm 1,81$ & $5,71 \pm 2,62$ & 0,03 & $6,08 \pm 1,83$ & $4,25 \pm 3,04$ & 0,36 \\
\hline Dor mais leve (24h) & $5,14 \pm 2,34$ & $2,85 \pm 2,41$ & 0,36 & $3 \pm 1,53$ & $1,91 \pm 2,10$ & 0,20 \\
\hline Média de Dor & $7,14 \pm 2,34$ & $5 \pm 2,38$ & 0,04 & $4,75 \pm 1,48$ & $3,5 \pm 2,71$ & 0,17 \\
\hline Dor no momento & $5,85 \pm 2,79$ & $2,14 \pm 3,18$ & 0,71 & $3,66 \pm 2,01$ & $1,66 \pm 2,38$ & 0,96 \\
\hline Alívio por analgésicos & $4,42 \pm 2,14$ & $4 \pm 2$ & 0,07 & $5,66 \pm 2,26$ & $6 \pm 1,77$ & 0,52 \\
\hline Interferência em: Atividades gerais & $8,71 \pm 1,49$ & $3 \pm 4,04$ & 0,58 & $6,5 \pm 2,61$ & $2,66 \pm 2,41$ & 0,31 \\
\hline Humor & $4 \pm 4,04$ & $2 \pm 2,88$ & 0,02 & $7,54 \pm 1,63$ & $2,58 \pm 2,84$ & 0,38 \\
\hline Caminhada & $7 \pm 3,41$ & $4,42 \pm 3,99$ & 0,65 & $5,75 \pm 2,34$ & $2,66 \pm 3,28$ & 0,65 \\
\hline Trabalho & $6,85 \pm 3,07$ & $4,42 \pm 3,30$ & 0,96 & $6,5 \pm 2,46$ & $3,16 \pm 2,69$ & 0,31 \\
\hline Relacionamento & $5 \pm 4,86$ & $1 \pm 1,91$ & 0,92 & $5,33 \pm 3,39$ & $1,58 \pm 2,02$ & 0,38 \\
\hline Sono & $8,85 \pm 1,67$ & $3,28 \pm 4,34$ & 0,71 & $6,41 \pm 3,98$ & $2 \pm 2,33$ & 0,65 \\
\hline Habilidades de apreciar a vida & $7,57 \pm 2,87$ & $2,14( \pm 3,93)$ & 0,49 & $6,25 \pm 3,41$ & $2,91 \pm 3,02$ & 0,44 \\
\hline
\end{tabular}

*GMEND = Grupo Meditação e Neurociência da Dor; ${ }^{+}$GM = Grupo Meditação

Tabela 4 - Análise intragrupo do GMEND* e GM+ para as avaliações pré e pós-intervenção. São Carlos, 2020

\begin{tabular}{|c|c|c|c|c|c|c|c|}
\hline Variáveis do GMEND* & Avaliação & Reavaliação & 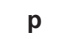 & Variáveis do GM"III & Avaliação & Reavaliação & $\mathbf{p}$ \\
\hline Pontos de dor & $40,42 \pm 5,12$ & $11 \pm 8,28$ & 0,01 & Pontos de dor & $36,91 \pm 11,01$ & $13,16 \pm 11,91$ & 0,00 \\
\hline Pior dor & $8,57 \pm 1,81$ & $5,71 \pm 2,62$ & 0,12 & Pior dor & $6,08 \pm 1,83$ & $4,25 \pm 3,04$ & 0,05 \\
\hline Dor + leve & $5,14 \pm 2,34$ & $2,85 \pm 2,41$ & 0,01 & Dor + leve & $3 \pm 1,53$ & $1,91 \pm 2,10$ & 0,24 \\
\hline Media dor & $7,14 \pm 2,34$ & $5 \pm 2,38$ & 0,12 & Média de dor & $4,75 \pm 1,48$ & $3,5 \pm 2,71$ & 0,18 \\
\hline Dor momento & $5,85 \pm 2,79$ & $2,14 \pm 3,18$ & 0,06 & Dor momento & $3,66 \pm 2,01$ & $1,66 \pm 2,38$ & 0,06 \\
\hline Alivio analg ${ }^{\dagger}$ & $4,42 \pm 2,14$ & $4 \pm 2$ & 0,34 & Alivio analg ${ }^{\dagger}$ & $5,66 \pm 2,26$ & $6 \pm 1,77$ & 0,06 \\
\hline Int ativ ${ }^{\ddagger}$ & $8,71 \pm 1,49$ & $3 \pm 4,04$ & 0,02 & Int ativ $v^{\ddagger}$ & $6,5 \pm 2,61$ & $2,66 \pm 2,41$ & 0,01 \\
\hline Int humorई & $4 \pm 4,04$ & $2 \pm 2,88$ & 0,28 & Int humor & $7,54 \pm 1,63$ & $2,58 \pm 2,84$ & 0,03 \\
\hline Int caminh $\|$ & $7 \pm 3,41$ & $4,42 \pm 3,99$ & 0,07 & Int caminh ${ }^{\|}$ & $5,75 \pm 2,34$ & $2,66 \pm 3,28$ & 0,02 \\
\hline Int trabalho" & $6,85 \pm 3,07$ & $4,42 \pm 3,30$ & 0,17 & Int trabalho" & $6,5 \pm 2,46$ & $3,16 \pm 2,69$ & 0,00 \\
\hline Int relac $c^{\star *}$ & $5 \pm 4,86$ & $1 \pm 1,91$ & 0,06 & Int relac ${ }^{\star *}$ & $5,33 \pm 3,39$ & $1,58 \pm 2,02$ & 0,01 \\
\hline Int sono ${ }^{\dagger \dagger}$ & $8,85 \pm 1,67$ & $3,28 \pm 4,34$ & 0,02 & Int sono ${ }^{\dagger \dagger}$ & $6,41 \pm 3,98$ & $2 \pm 2,33$ & 0,00 \\
\hline Int hab & $7,57 \pm 2,87$ & $2,14 \pm 3,93$ & 0,06 & Int hab & $6,25 \pm 3,41$ & $2,91 \pm 3,02$ & 0,13 \\
\hline$F I Q \S \S$ & $69,42 \pm 8,75$ & $31,95 \pm 18,94$ & 0,01 & $F / Q \S \S$ & $55,44 \pm 13,01$ & $33,42 \pm 20,85$ & 0,00 \\
\hline Ansiedade & $8,57 \pm 1,90$ & $5,85 \pm 3,80$ & 0,15 & Ansiedade & $10,75 \pm 3,72$ & $7,33 \pm 4,03$ & 0,00 \\
\hline Depressão & $7,85 \pm 5,52$ & $3 \pm 3,55$ & 0,10 & Depressão & $8,5 \pm 3,23$ & $6,41 \pm 3,72$ & 0,02 \\
\hline Catastrofização & $27,28 \pm 9,46$ & $12,85 \pm 9,80$ & 0,01 & Catastrofização & $23,66 \pm 12,69$ & $11,33 \pm 9,34$ & 0,00 \\
\hline Cinesiofobia & $43 \pm 7,34$ & $43,71 \pm 10,59$ & 0,75 & Cinesiofobia & $41,36 \pm 9,03$ & $43,66 \pm 8,95$ & 0,12 \\
\hline Sono & $13,42 \pm 2,57$ & $6,57 \pm 3,69$ & 0,01 & Sono & $11,41 \pm 5,45$ & $7,08 \pm 4,39$ & 0,00 \\
\hline
\end{tabular}

*GMEND = Grupo Meditação e Neurociência da Dor; ${ }^{\dagger}$ Alivio Analg = alívio por analgésico; ${ }^{\text {Int }}$ ativ = interferência da dor nas atividades gerais; ${ }^{\circledR}$ Int humor = interferência da dor no humor; "Int caminh = interferência da dor na caminhada; "Int trabalho = interferência da dor no trabalho; **Int relac = interferência da dor nos relacionamentos; ${ }^{+\dagger}$ Int sono = interferência da dor no sono; ${ }^{\ddagger}$ Int hab= interferência na habilidade de apreciar a vida; ${ }^{\S \S} \mathrm{FIQ}=$ Questionário de Impacto da Fibromialgia; "IIGM= Grupo Meditação

\section{Discussão}

O presente estudo teve por objetivo analisar os efeitos da terapêutica baseada no método Mindfulness com associação a Educação em Neurociências da Dor na interferência da dor, capacidade funcional, humor e sono de adultos e idosos com Fibromialgia de uma cidade do interior paulista. Para tanto, a pesquisa contou com a análise longitudinal e quantitativa de dois grupos de intervenção: o grupo GM (Grupo Meditação) e o grupo GMEND (Grupo Meditação e Educação em Neurociências da Dor). Os resultados obtidos sugerem que ambas as intervenções têm impacto positivo na sintomatologia da fibromialgia, capacidade funcional, níveis de humor e sono de pessoas acometidas por essa doença.

Os resultados das comparações intra-grupos sugerem que as intervenções propostas beneficiaram os participantes com melhorias na maioria das variáveis estudadas. A intervenção baseada em Mindfulness associada à educação em neurociência da dor demonstrou ser eficiente para reduzir a percepção da dor, a interferência da mesma nas atividades gerais e no sono, no questionário de impacto da fibromialgia 
na catastrofização e na qualidade do sono. Já o grupo que participou apenas da intervenção baseada em Mindfulness apresentou não apenas a melhora nas variáveis citadas para o grupo anterior, como também melhorou na pontuação dos sintomas de ansiedade e depressão.

Não foram observadas diferenças significativas após as intervenções quando os grupos foram comparados. Contudo, como foram observadas melhoras nas análises intragrupos, podemos inferir que, de um modo geral, as intervenções proporcionam benefícios, mas seus efeitos não diferem entre si.

O Mindfulness atualmente tem ganhado destaque no âmbito das neurociências por suas contribuições, dentre elas a de nível cerebral (29-30). As pesquisas que envolvem intervenções baseadas em Mindfulness têm mostrado consideráveis melhorias nos níveis de insônia, depressão, ansiedade e manejo da fibromialgia, o que sugere um papel importante em teorias cognitivas do sono e em comportamentos mal adaptativos em relação a dor ${ }^{(15,30-31)}$. Em consonância com a literatura, nosso estudo demonstrou tais benefícios.

Estudos que mostram o impacto das terapias baseadas em Mindfulness nos sintomas físicos e psicológicos da fibromialgia evidenciam que os níveis de atenção estão associados a menores impactos da doença, o que pode se desdobrar em resultados positivos nas variáveis sono, relacionamento com a dor e relacionamento com doença( ${ }^{(32)}$. Apesar de não ter sido utilizada uma escala específica para a avaliação da atenção dos participantes, este estudo demonstrou que os mesmos tiveram não apenas a melhoria no sono, como também na percepção da interferência da dor nos relacionamentos, bem como na percepção da dor propriamente dita.

Além disso, pesquisadores (33) ressaltam a importância da elaboração de mecanismos e abordagens bem estruturados, detectando fatores influenciáveis em comum e como a associação da prática atrelada a outras terapias podem ter resultados distintos. A frequência de práticas e afinidade também são dignas de nota pois podem influenciar diretamente nos resultados que a prática pode oferecer(11,15,32-33). Estas considerações justificam a descontinuidade dos participantes que tiveram faltas acima dos limites pré-estabelecidos.

A literatura reconhece que um tratamento biopsicossocial para dor opta por usar habilidades cognitivas, emocionais e comportamentais, compreendida por estratégias integrais que atendem os níveis de complexidade da dor e um olhar holístico ao paciente, contribuem para a redução da dor e sofrimento(11). Por isso, considerando a perspectiva de que a fibromialgia apresenta múltiplos fatores influenciando de dor, estratégias como END vem ganhando reconhecimento para atenuar efeitos psicológicos e também físicos da doença. Apesar da literatura evidenciar melhoras nos quadros de dor, incapacidade e níveis de catastrofização ainda se questiona a diferença de resultados a partir das diferentes doses aplicadas. Neste estudo, a intervenção com neurociência da dor, para o GMEND, ocorreu por três semanas e não observamos diferenças significativas quando comparamos com o GM. Estudos sugerem que dosagens mais elevadas podem conduzir a uma diminuição na intensidade da dor em pacientes com fibromialgia acompanhados ao longo de três meses ${ }^{(34-35)}$.

A END aplicada isoladamente( ${ }^{(35)}$ não demonstra uma intervenção viável para a modificações dessas variáveis. Os autores salientam a importância do modo em que consiste a aplicação da técnica, que deve envolver um clínico especializado e propiciar um treinamento ao provedor da educação, o que resultaria em uma melhor experiência ao paciente. Por este motivo, este trabalho de propôs a aplicar tal intervenção em conjunto com a prática da intervenção baseada em Mindfulness.

Observamos em nosso estudo a descontinuidade de alguns participantes, que relataram a não adaptação pela prática de Mindfulness. Neste sentido, uma melhor adesão e engajamento para os que tem afinidade para a prática de meditação auxilia esses indivíduos a se manterem nos programas de intervenção(33). Segundo os autores, isso proporciona uma melhor adaptação da prática no dia a dia dos participantes.

Os sentimentos e desconexão e fragmentação que muitas vezes acompanha o sofrimento, e o encorajamento para a libertação das prisões de nossos próprios hábitos e pensamentos, que engloba mudanças nos hábitos de vida e a intenção de cultivar intencionalmente a compaixão por toda a vida, predita nas bases dos mecanismo de meditação voltadas para compaixão, salientando melhorar sentimentos de conexão, pertencimento e padrões menos rígidos ${ }^{(33)}$.

Como resultados adicionais, a vivência da prática em grupo propiciou maior compreensão e acolhimento entre os indivíduos, o que pode contribuir para maior compartilhamento de experiências, sociabilidade e reflexões sobre o cotidiano dos participantes, propiciando a vivência de descrição dos sentimentos e emoções e aceitação coletiva.

Como limitação, cita-se a não avaliação das multifaces do Mindfulness, o que pode ser importante para a compreensão integral do quadro do paciente e atender as possíveis dificuldades que ele pode encontrar com a técnica. Além disso, não foi avaliado o nível de atenção dos participantes. Por fim, a dificuldade de locomoção até o local das práticas pode ter sido uma barreira aos participantes. 


\section{Conclusão}

O presente estudo teve por objetivos analisar os efeitos da terapêutica baseada em Mindfulness, e se associada a Educação em Neurociências da Dor, que por sua vez consiste em reconceituar a dor e através da exposição do conhecimento sobre a neurobiologia da dor, em pacientes com fibromialgia no interior paulista. Como resultados, pudemos perceber que a técnica meditativa possui boa eficácia atenuando o quadro sintomatológico da dor crônica, na capacidade funcional e sintomas psicológicos. Embora a literatura aponte os efeitos benéficos da educação em neurociências da dor sobre os pensamentos catastróficos e cinesiofobia, nesta pesquisa não foi possível notar diferenças estatisticamente significantes nas análises intergrupos. Contudo, observou-se melhoras significativas nas análises intragrupo para ambas as intervenções, o que sugere que tanto a intervenção Mindfulness quanto a associação do Mindfulness com neurociência da dor pode ser benéfica para pacientes com fibromialgia.

\section{Agradecimentos}

Agradecemos a Patrícia Santos por ministrar as práticas de mindfulness.

\section{Referências}

1. Neri AL. Palavras-chave em gerontologia. $4^{a}$ ed Campinas: Alínea; 2017.

2. Rivero T, Canali-Prado F, Vieira V, Rivero A. Aspectos psicossociais do envelhecimento. In: MalloyDiniz L, Fuentes D, Cosenza R. Neuropsicologia do envelhecimento: uma abordagem multidimensional. Porto Alegre: Artmed; 2013. p. 64-77.

3. Lebrão ML. O envelhecimento no Brasil: aspectos da transição demográfica e epidemiológica. Saúde Coletiva. 2007; 4(17):135-40.

4. Dellaroza MSG, Pimenta CAM, Matsuo T. Prevalência e caracterização da dor crônica em idosos não institucionalizados. Cad Saúde Pública. 2007;23(5):1151-60. http://dx.doi.org/10.1590/ S0102-311X2007000500017.

5. Vasconcelos FH, Araújo GC. Prevalence of chronic pain in Brazil: a descriptive study. BrJP, 2018; 1(2):176-179. 6. Kreling MCGD, Cruz DALM, Pimenta CAM. Prevalencia de dolor cronico en adultos. Rev Bras Enferm. 2006; 59(4):509-13. doi: http://dx.doi.org/10.1590/ S0034-71672006000400007.

7. Sá K, Baptista AF, Matos MA, Lessa I. Prevalência de dor crônica e fatores associados na população de Salvador, Bahia. Rev Saúde Pública. 2009; 43(4):622-30. 8. Andrew R, Derry S, Taylor RS, Straube S, Phillips CJ. The costs and consequences of adequately managed chronic non-cancer pain and chronic neuropathic pain. Pain Practice. 2014;14(1):79-94. doi: 10.1111/ papr.12050. Epub 2013 Mar 6.

9. Mills SEE, Nicolson KP, Smith BH. Chronic pain: a review of its epidemiology and associated factors in population-based studies. $\mathrm{Br}]$ Anaesthesia. 2019;123(2):273-83. doi: 10.1016/j.bja.2019.03.023. 10. Dellaroza MSG, Furuya RK, Cabrera MAS, Matsuo T, Trelha C, Yamada KN, Pacola L, et al. Caracterização da dor crônica e métodos analgésicos utilizados por idosos da comunidade. Rev Assoc Med Bras. 2008; 54(1)36-41. 11. Darnall BD. Psychological treatment for patients with chronic pain. Washington, DC: American Psychological Association; 2019. doi: https://doi. org/10.1037/0000104-000

12. Celich KLS, Galon C. Dor crônica em idosos e sua influência nas atividades da vida diária e convivência social. Rev Bras Geriatria Gerontol. 2009; 12(3):345-59. 13. Dellaroza MSG, Pimenta CADM, Duarte YA, Lebrão ML. Dor crônica em idosos residentes em São Paulo, Brasil: prevalência, características e associação com capacidade funcional e mobilidade (Estudo SABE). Cad Saúde Pública. 2013; 29(2):325-34. doi: https://doi. org/10.1590/S0102-311X2013000200019.

14. Gui M, Pedroni CR, Rossini S, Reimão R, Barbosa CMR. Distúrbios do sono em pacientes com fibromialgia. Neurobiologia. 2010; 73(1):175-82.

15. Adler-Neal AL, Zeidan F. Mindfulness meditation for fibromyalgia: mechanistic and clinical considerations. Curr Rheumat Rep 19; 2017; 19(9). https://doi. org/10.1007/s11926-017-0686-0

16. Marques AP, do Espírito Santo ADS, Berssaneti AA, Matsutani LA, Yuan SLK. Prevalence of fibromyalgia: literature review update. Rev Bras Reumatol. 2017;57(4):356-63. doi: 10.1016/j.rbre.2017.01.005

17. Marchiori MFR. Estudo dos efeitos fisiológicos e psicológicos de uma técnica de Meditação Zen em uma amostra randomizada de idosos hipertensos. [Dissertação Mestrado]. São Paulo: Escola Paulista de Medicina, Universidade Federal de São Paulo; 2012. 107 p.

18. Goleman D. A arte da meditação: um guia para a meditação. Rio de Janeiro: Sextante; 1999.

19. Demarzo MMP. Meditação aplicada à saúde. Programa de Atualização em Medicina de Família e Comunidade. Porto Alegre: Artmed; 2011. p. 6:1.

20. Lifshitz M, Thompson E. What's wrong with "the mindful brain"? Moving past a neurocentric view of meditation. In: Casting Light on the Dark Side of Brain Imaging. Califórnia; Montreal: Academic Press; 2019. p. $123-8$.

21. Redondo FCS, Valle AC. Meditação: Novas Perspectivas Terapêuticas Para Controle Da Dor Crônica. Nanocell News. 2015;3(2), nov 2015. doi: http://dx.doi. org/10.15729/nanocellnews.2015.11.03.002 
22. Fancourt D, Steptoe A. Physical and Psychosocial Factors in the Prevention of Chronic Pain in Older Age. J Pain. 2018;19(12):1385-91. doi: 10.1016/j. jpain.2018.06.001

23. Malfliet A, Van Oosterwijck J, Meeus M, Cagnie B, Danneels L, Dolphens M, Nijs J. Kinesiophobia and maladaptive coping strategies prevent improvements in pain catastrophizing following pain neuroscience education in fibromyalgia / chronic fatigue syndrome : An explorative study. Physiother Theory Practice. 2017;33(8):653-60. doi: 10.1080/09593985.2017.1331481

24. Louw A, Zimney K, Puentedura EJ, Diener I. The efficacy of pain neuroscience education on musculoskeletal pain: a systematic review of the literature. Physiother Theory Practice. 2016;32(5):33255. doi: 10.1080/09593985.2016.1194646.

25. Moseley L. Combined physiotherapy and education is efficacious for chronic low back pain. Aust J Physiother. 2002;48(4):297-302. doi: 10.1016/ s0004-9514(14)60169-0.

26. Clarke CL, Ryan CG, Martin DJ. Pain neurophysiology education for the management of individuals with chronic low back pain: a systematic review and metaanalysis. Man Ther. 2011; 16(6):544-9. doi: 10.1016/j. math.2011.05.003

27. Louw A, Diener I, Butler DS, Puentedura EJ. The effect of neuroscience education on pain, disability, anxiety, and stress in chronic musculoskeletal pain. Arch Phys Med Rehabil. 2011;92(12): 2041-56. doi: doi:10.1016/j.apmr.2011.07.198

28. Kabat-Zinn J. Viver a catástrofe total: como utilizar a sabedoria do corpo e da mente para enfrentar o estresse, a dor e a doença. 2aed. São Paulo: Palas Athena; 2019. 700 p.

29. Moore A, Malinowski P. Meditation, mindfulness and cognitive flexibility. Consciousness Cognition. 2009;18(1):176-86. doi:10.1016/j.concog.2008.12.008 30. Tang YY, Hölzel BK, Posner MI. The neuroscience of mindfulness meditation. Nature Rev Neurosci. 2015;16(4):213. doi: https://doi.org/10.1038/nrn3916 31. Schneider MN, Zavos HM, McAdams TA, Kovas Y, Sadeghi S, Gregory AM. Mindfulness and associations with symptoms of insomnia, anxiety and depression in early adulthood: a twin and sibling study. Behav Res
Ther. 2019;118:18-29. doi: https://doi.org/10.1016/j. brat.2018.11.002

32. Pleman B, Park M, Han X, Price LL, Bannuru RR, Harvey WF, Wang $C$. Mindfulness is associated with psychological health and moderates the impact of fibromyalgia. Clin Rheumatol. 2019:1-9. doi: http:// dx.doi.org/10.1007/s10067-019-04436-1

33. Rosenkranz MA, Dunne JD, Davidson RJ. The next generation of mindfulness-based intervention research: what have we learned and where are we headed? Curr Opinion Psychol. 2019; 2:193-83. doi: https://doi. org/10.1016/j.copsyc.2018.12.022.

34. Cuenca JJA, Pecos-Martín D, Martínez-Merinero P, Lluch Girbés E, Nijs J, Meeus M, Fernández-Carnero J. How Much Is Needed? Comparison of the Effectiveness of Different Pain Education Dosages in Patients with Fibromyalgia. Pain Med. 2020;21(4):782-93. doi: https://doi.org/10.1093/pm/pnz069

35. Watson JA, Ryan CG, Cooper L, Ellington D, Whittle $R$, Lavender $M$, Martin DJ. Pain neuroscience education for adults with chronic musculoskeletal pain: a mixedmethods systematic review and meta-analysis. J Pain. 2019;20(10): 1140.e1-1140.e221140. doi:https://doi. org/10.1016/j.jpain.2019.02.011.

\section{Contribuição dos autores}

Concepção e planejamento do estudo: Lucas Pelegrini Nogueira de Carvalho, Lílian Silva Araújo, Maria do Perpétuo Socorro Lage Avelar e Karina Gramani Say. Obtenção dos dados: Lílian Silva Araújo e Maria do Perpétuo Socorro Lage Avelar. Análise e interpretação dos dados: Lucas Pelegrini Nogueira de Carvalho, Lílian Silva Araújo e Karina Gramani Say. Análise estatística: Lílian Silva Araújo e Karina Gramani Say. Redação do manuscrito: Lucas Pelegrini Nogueira de Carvalho, Lílian Silva Araújo e Karina Gramani Say. Revisão crítica do manuscrito: Lucas Pelegrini Nogueira de Carvalho, Maria do Perpétuo Socorro Lage Avelar e Karina Gramani Say. Outros (Aplicação da técnica mindfulness): Maria do Perpétuo Socorro Lage Avelar.

Todos os autores aprovaram a versão final do texto.

Conflito de interesse: os autores declararam que não há conflito de interesse 